\title{
A Survey and Research Proposal of Plant Leaf Disease Diagnosis and Classification on Mobile Devices
}

\author{
T.Nagarathinam ${ }^{1}$, Dr. K. Rameshkumar ${ }^{2}$ \\ PhD Research Scholar ${ }^{1}$, PhD Research Guide ${ }^{2}$ \\ ${ }^{I}$ (Department of Computer Science, MASS College of Arts \& Science, Kumbakonam, Tamil Nadu, India) \\ ${ }_{2}^{2}$ (Department of Computer Science, MASS College of Arts \& Science, Kumbakonam, Tamil Nadu, India)
}

\begin{abstract}
A plant disease is defined as anything that prevents a plant from performing to its maximal potential. A plant disease has impacted society and world olden times. Plant diseases have caused enormous economic losses in each one the countries. Diagnosis of diseases in plant is the fortitude of the grounds of a disease in plant, and detection refers to the identification of microorganisms. Failures in disease diagnosis show the way openly too little disease control and restrain in crop production and quality, and as a result trade. In this survey we bring into play some effectual papers to obtain detailed investigation and propose our research work of plant disease detection and classification on mobile devices.
\end{abstract}

Keywords: Brown spot, Classification, Cluster analysis, Disease, Leaf Blight,

\section{Introduction}

A plant disease is defined as "anything that prevents a plant from performing to its maximal potential". This definition includes abiotic and biotic plant diseases. The abiotic diseases caused by conditional external to the plant. It cannot multiply from plant to plant. Examples for these types of diseases being nutritional deficiency, soil compaction, salt injuries and sun scorch. Biotic diseases caused by living organisms which are called plant pathogens when they infect plants. Pathogens can spread from plant to plant and may infect all types of plant tissues including leaves, shoots, stems, crowns, roots, tuber, fruits, seeds, and vascular tissues. Plant pathogens consist of fungi, organism, bacteria, viruses, phytoplasmas, viroids etc., three components are absolutely necessary for diseases to occur in any plant system which are, a susceptible host plant, a virulent pathogen, a favorable environment. The chain of events involves in disease development such as Inoculation, Penetration, infection, Incubation, Reproduction, and Survival [1]. Image processing and data mining are the promising technologies to discover the diseases in plants. In early developed application only use the mobile device for capturing the images of infected plant or preprocessing the images for features extraction using the data mining and image processing techniques. Then the image or features sent to the local server or centralized or cloud to classify the image to identify the types of disease that appear in the plant. It consist of plenty of difficulty such as need a consistent internet connectivity, image transmission cost, delay in getting suggestion etc., To root out these troubles the proposed application will help farmer for find the types of disease that appear in the plant in their handset itself without waiting for get result from the server ( classification done in the server). Image classification on limited devices such as cellular phone is difficult because its limited capability to process, and storage using various classifier such as SVM, PNN etc., Hence we have propose a solution of these problem using cluster analysis technique which does not require any pre- training for classify the image and also it require limited memory to store the data. The paper has been arranged in section -I for plant introduction. In section- II illustrate the exiting research work for disease detection. At section - III portray summary of the existing method for quick references. The section- IV shows the current research proposal and final section fulfilled with conclusion.

\section{1. Off-device Image Processing Approaches}

\section{Existing Research Works}

Researchers started learning the matter of image processing on mobile devices. Owing to the limited computing capacity on mobile phones and PDAs, there are diverse approaches to dealing with this issue, of which the most significant will be server-client based system. The user captures an image using mobile device, which is next sent to a server that bring out the actual processing work. Later than processing, the respond is sent back to the user via the mobile phone networks .In this approach only the mobile device can be used to capture the plant image, sending the image to the server, this require high cost and time to transfer the image to the server. The below literature survey exhibit this type of approach. In this thesis [2] the authors' describes developing an android application to engender an automated mobile-based system to detect diseases of potato leaves diseases (table -1) which are "Early Blight" and "Late Blight" without fetch the expert to the field. 
A Survey and Research Proposal of Plant Leaf Disease Diagnosis and Classification on Mobile..

Table - 1: The equations are tabulated as follows

\begin{tabular}{|l|c|c|c|}
\hline \multirow{2}{*}{ Equation no } & Logical components & \multicolumn{2}{|c|}{ Trtion } \\
\cline { 3 - 4 } & & Flob is round & Blob is not round \\
\hline 1 & Roundedness (R) & Blob is Brown & Blob is Purple \\
\hline 2 & Color (C1) & Blob is centric ring is found & Blob is not centric ring is found \\
\hline 3 & Centric Ring (Cr) & Blob is limited by the vein & blob is not limited by the vein \\
\hline 4 & Leaf Vein (L) & Diameter of the blob is $1 / 8$ inch & $1 / 8$ inch \\
\hline 5 & Diameter (D) & & \\
& &
\end{tabular}

They use "Mathematical morphology", "Leaf Vein Detection" and "Blob detection" algorithms in MATLab to detect the diseases. Once the input gathered from the users, it could be sent to the online server for execution. MATlab in server generate the output and the android parsed the result through PHP command to send it to the user. In this application, "Blob detection" algorithm detects the diseases region using two types of images namely RGB image and Binary image. Finally the author calculates the precision, recall and accuracy using the formula - (2), (3) and (4) respectively.
Precision calculation
$: t p / t p+f p$
Recall calculation
$: t p t p+f n$
Accuracy calculation
$: t p / t p+t n+f p+f n$

The pictorial representation of the above literature survey is as follows fig -1 .

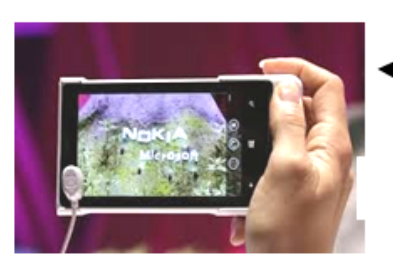

MOBILE : :Image capturing

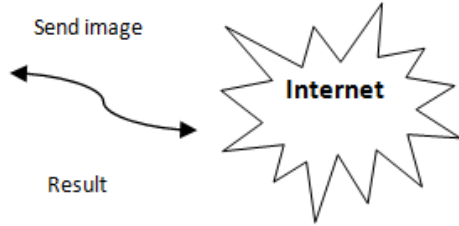

Fig-1

\subsection{On-device segmentation Approaches}

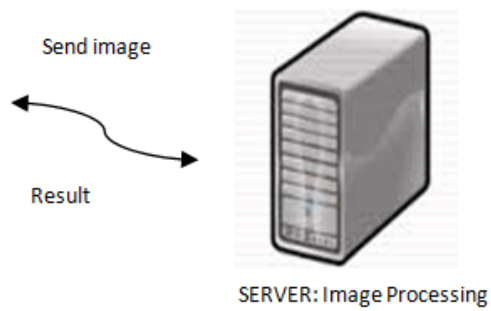

In this approach the image capturing, pre-processing and segmentation done on the mobile devices, the segmented image portion send to the server for further classification and diseases identification. The following literature survey exhibit the approach. In "Multi-resolution mobile vision system for plant leaf disease diagnosis" [7] the authors Shitala Prasad, Sateesh K. Peddoju and Debashis Ghosh proposed a mobile clientserver architecture for Plant leaf disease detection and diagnosis using a novel combination of Gabor wavelet transform (GWT) and gray level co-occurrence matrix (GLCM). The system is a symbol of diseased patch in multi-resolution and multi-direction feature vector. The proposed system consists of two modules such as Mobile Client and Pathology Sever. In the first module, the images of the plant leaf has captured by mobile camera and pre-processes the leaf image, segments diseased patches in the mobile device itself and the segmented portion is transmits to the Pathology Sever for diseases detection. In the second module, Pathology Sever, receive the segmented portion of the image and extract the features using a novel combination of Gabor Wavelet Transform (GWT) and Gray Level Co-occurrence Matrix (GLCM), finally the classifier k-Nearest Neighbor classifies the segmented portion to diagnosis the disease that appear in the portion of segmentation. Once the disease has been identified that it is sends back to the user mobile (mobile client) via an SMS with an accuracy rate of $93 \%$. The proposed approached identifies leaf spots and leaf blotch diseases on the plant leaf and it is tested in Mobile devices namely, Android phones or tablets. The diagrammatical representation of the survey is depicted as follows fig-2.
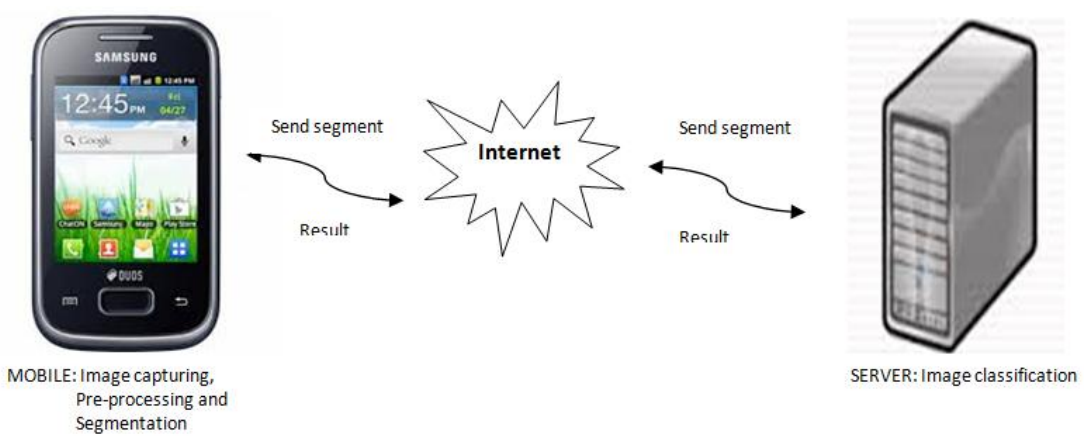

Fig-2 


\subsection{On-device image Features extraction Approaches}

In this approach describes the issue of image processing on mobile devices. One of the solutions is the use of a server-client based system. The user captures an image with the mobile device, which is then preprocessed in mobile device itself (features extraction) and the extracted features sent to the server that carries out the actual processing work (classification). After classification, the reply is sent back to the user via the mobile phone network. The major advantage is that the task can be carried out in both mobile phone and server. Hence the features only sent to the server data transfer time and cost significantly reduced by the farmer. However, this system requires a constant network connection to be available, which can be difficult in certain areas or inside buildings. The survey of following might exhibit the approach uses.

In paper [3] the authors developed an application for smart phone to identify fungal leaf spots diseases on Sugar beet by analyzing digital images with minimal computation cost and have no accessing expert knowledge. In this application the captured image are stored in an image database and it has been classified by experts to training a classification algorithm. In their proposed application captured image has been preprocessed on Smartphone before it sent to the local server for further analysis. The preprocessing of image has starting up with down-sample the input image and max RGB filter has applied to identifies the reddish or brownish regions from the image and then binary image is computed to put the reddish pixels in the foreground and greenish / bluish pixels in the background. Region image has computed by using various filtering method such as median filtering, hole filling etc., finally the preprocessed resulting image inputted to the local server to extract the features of each region the LBP of the gradient magnitudes were computed and binned into a histogram to use its entropy as a descriptor. Naïve Bayer classifier attaches the regions to predefined classes (done by experts). The authors finally bring to a close the result of the application disease recognition accuracy of more than $97 \%$. The application can detect the three diseases ( Cerospora beticola, Ramularia, Phoma betae) on sugar beet. The main benefit of the developed application reduces the computation cost and speed up the processing time due to classification done in local server instead of Smartphone. Even if it has the benefit, it also has some limitation which are it require constant internet connection to transferring the features from smart phone to local server for classification and it need some extra software for both the client and server side.

In paper [4] the author Shovon Paulinus Rozario exhibit 'Krishokbondhu', an automated system for the farmers to make out four types of paddy diseases using their mobile phones. In this thesis he used computer vision techniques to detection of affected spots from the mobile captured image and their classification. The disease affected portions were segmented by color approach and the features (based on blobs, area and color) were extracted from the segmented images using Blob detection algorithm. Classification was done by using the features. In his research he used the binary search tree to mapping the feature values for comparison of Euclidean distance during the classification. A mobile captured image has been send to the remote server for processing and classification via the internet connectivity. Once the process (noise removal by bilateral smoothing filter, segmentation done by color feature based approach, etc.,) has over in the server then the expert analyze the processed results and give advice to the farmers to their mobile phones. In this developed application could identifies four types of diseases in crops such as Bacterial Leaf Blight, Brown Spot, Leaf Blast and Leaf Scald these has been tabulated as in the following using the equation (1).

$$
\text { Accuracy }=\mathrm{TR} / \mathrm{TR}+\mathrm{FR}
$$

Where

$$
\begin{aligned}
& \text { TR - True Recognition } \\
& \text { FR - False Recognition }
\end{aligned}
$$

Table- 2. Shows the Diseases and its accuracy is tabled as follows

\begin{tabular}{|c|c|c|c|}
\hline Disease Name & TR & FR & Accuracy (\%) \\
\hline Bacterial Leaf Blight & 7 & 2 & 77.77 \\
\hline Brown Spot & 29 & 3 & 90.62 \\
\hline Leaf Blast & 17 & 1 & 94.44 \\
\hline Leaf Scald & 11 & 1 & 91.66 \\
\hline Healthy paddy & 23 & 23 & 100 \\
\hline
\end{tabular}

In [5], develops an application (AgroMobile) utilizing Mobile Cloud Computing on handsets for farmers to better cultivation and marketing their agricultural products. Since this framework uses MCC to solve the various issues in both developer and user (farmer) sides, which are it beat the margins of mobile processing power, data storage, assist make longer the battery time by moving the computations on cloud server, and augment the protection of all mobile devices and boost mobility. They proposed a conceptual model of MCC technology for handheld device named as "Agrocloud". In their model, AgroMobile server provides SaaS using Application Service Provider in the cloud, to the users. The developer is connected to the ASP and the end users (smartphone, tablet, laptop, or phablet with $2.5 \mathrm{G}, 3 \mathrm{G}$ or $4 \mathrm{G}$ technologies) are connected to AgroMobile 
infrastructure providing application services. The task can be done on the two places, one in mobile device itself which is image pre-processing (noise removal, normalization, image equalization, and few morphological operations), after a button click. Within few seconds the result is revert back from AgroMobile to the mobile device. For machine learning algorithm support vector machine (SVM) is used with Radial base function (RBF) kernel, the following equation (1),

$$
\begin{gathered}
\left(x, x^{\prime}\right)=e-R \| x-\left.x^{\prime}\right|^{2} \text { for all } R>0 \\
\text { Where }
\end{gathered}
$$

$R$ determines the area of influence over the feature space. RBF kernel adds bumps to the low dimension features to represent it in the high dimension feature space. The authors use tools like OpenNebula 2.0, and MATLAB 2012b for experimental setup and an Android based mobile devices of version 2.3.5 and above with a processing speed of $860 \mathrm{MHz}$ and above are used for designing and testing. Their developed application provides some following services for the farmers which are, obtain detailed information and SMS, Audio and Video facility to narrate the illiterate farmer, and input their queries to obtain reply with their native language. Even if the application offer the services, it has some demerit such as require internet $\mathrm{Wi}-\mathrm{Fi}$ connection and it only work in the campus since it uses Wi-Fi facility.

The authors in paper [6] present an inventive thought concerning diseases affected by paddy crops and provide the elucidation to disease using mobile devices for farmer in rural areas. In their proposed method consist of five functionalities such as Image capture, Image selection, Image zoom and crop, Share image with expert group and Receive notification from the central server. In the first stage the image has captured by using mobile phone. At second stage, if the image has been already stored in the mobile device that it has to be selected from the photo library of the device. In third stage, the application in the mobile allows zoom the affected region of paddy using pinch with two fingers then the disease affected image and a unique URL uploaded in the remote server using HTTP. The experts in the server side examine the image and uncover the types of diseases that appear in the crop and suggestion of the experts send to the client application in the mobile device in the form of SMS/MMS. The pictorial representation of the above literature survey is as below fig-3

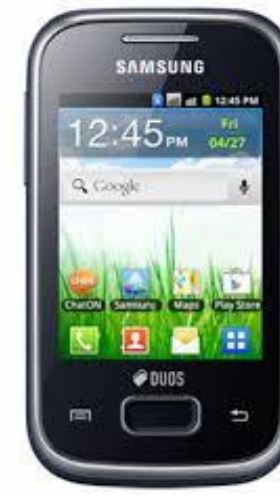

MOBILE: Image capturing \& Pre-processing
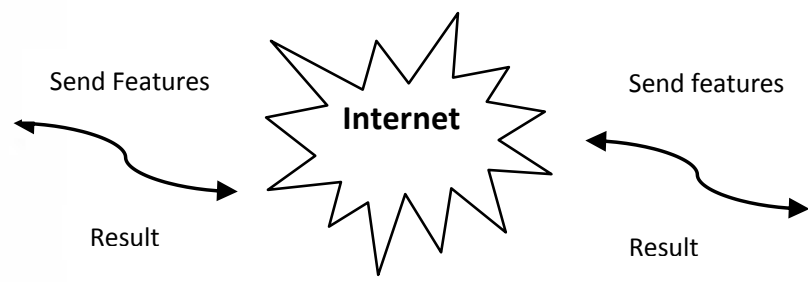

Fig-3

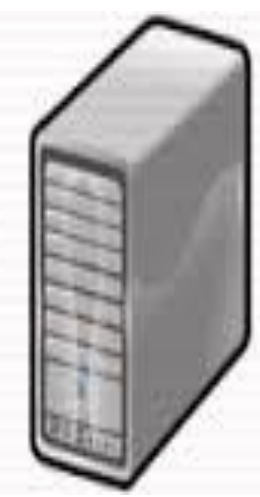

SERVER: Image classification

\subsection{On-device Image Processing Approaches}

In this approaches the image processing done on the mobile device itself without sending the image or features to the server for pre processing and classification. In this approach, image can be capturing, preprocessing and classification done on the mobile device itself. Hence the farmer no needs to have internet connection, transferring of image or features, image transfer cost. The following literature survey shows this approach.

In paper[8] the authors Rahat Yasir and Nova Ahmed describes the development of application software has named as "Beetles", to support rural people for detecting crop diseases in real time using mobile device with limited computing capabilities and storage space; without need of internet connectivity. To develop such an application they use two algorithms namely Histogram and $\mathrm{YC}_{b} \mathrm{C}_{\mathrm{r}}$. After the image capturing is over, the histogram algorithm employed to found intensity value of each pixel in the image and it is showed in graph. Then calculate the Histogram value using following formula.

$$
=\text { Total number of pixels / total number of pixel at the intensity. }
$$


In such a way various steps processed to obtain 10 different values which are stored in an array. Since this array the authors make use of 6 values as the unique id for each crop diseases and it would compared with the stored unique id. At this time $\mathrm{YC}_{b} \mathrm{C}_{\mathrm{r}}$ algorithm employing for found the exact color code of the leaf. If the matching rates of those ids have above $80 \%$ and the color code of the infected crop matched with the stored color codes then it shows the successfulness of disease detection and replies the disease name and cure steps to the Farmers / users. The authors describes that the application can detect six types of diseases, which are brown leaf spot, bacterial leaf blight, brown spot, Urfa and rice blast, that affect the crop, Finally the authors show the performance of the detection algorithms were tabulated in the following table- 3 .

Table- 3. Shows Diseases and its accuracy is tabulated as above.

\begin{tabular}{|l|c|c|c|}
\hline \multicolumn{1}{|c|}{ Disease name } & $\mathbf{Y C}_{\mathbf{b}} \mathbf{C}_{\mathbf{r}}(\mathbf{S})$ & Histogram $(\mathbf{S})$ & Detection rate \\
\hline Narrow Brown leaf spot & 2.05 & 1.40 & $93 \%$ \\
\hline Brown spot & 2.66 & 1.32 & $89 \%$ \\
\hline Bacterial Blight & 2.34 & 1.56 & $86 \%$ \\
\hline Healthy leaf & 1.30 & 1.15 & $98 \%$ \\
\hline
\end{tabular}

Finally, the authors concluded the over all disease detection rate of the application has been more than $85 \%$. According to the authors, that the software can display the disease type and cure steps in native language of the Bangladesh farmers and it narrate the disease type in their native language. Since this review of the paper we can conclude that the application has around $15 \%$ possible to failure of overall disease detect rate. The system fails/ less to detecting diseases in the image if that it is captured in poor lighting condition because the color code used to compare for discover the diseases.

In [9] proposes application of image processing and machine learning in recognize three palm oil diseases based on visual manifestation. They were design a method with linear complexity process in order to minimize processing time so that it can be implemented in mobile device. Their developed application by means of image processing to dig out six types of features (based on leaf spot color and shape) which are Median of RGB, Quartile 1 of RGB, Quartile 3 of RGB, Average Brightness using the equation (1), Standard deviation of RGB using the equation (2) and Image shape using the equation (3) from palm leaf image then the pattern was learned using Neural Network method in machine learning process. As of the learning process they yield a classification model by $87.75 \%$ average accuracy and then a mathematical equation (4) being formulated based on classification model in order to be implemented in mobile device.

$$
B=0.299 r+0.587 g+0.114 b \text {, }
$$

Where:

$$
\begin{array}{r}
\mathrm{rgb}=\text { mean of RGB value in all } \\
\qquad \sigma=\operatorname{sqrt}\left(\frac{\sum_{i=1}^{\mathrm{n}}(\mu-\mathrm{xi})}{\mathrm{n}}\right)
\end{array}
$$

Where:

$\mathrm{n}=$ the total contained pixel

$\mu=$ mean red, green or blue of all contained pixel, depends on what color is being calculated.

$\mathrm{x}=$ red, green or blue value of pixel, depends on what color is being calculated.

$$
\mathrm{S}=\mathrm{a} / \mathrm{L}
$$

Where:

$$
\begin{aligned}
& \mathrm{a}=\text { spot area size and } \mathrm{L}=\text { spot perimeter length } \\
& \text { Out }=\tan \operatorname{sig}(\mathrm{W} 2 \times(\operatorname{tansig}(\mathrm{W} 1 \times \text { input }+\mathrm{b} 1)+\mathrm{b} 2)
\end{aligned}
$$

Where: $\operatorname{tansig}=$ is a tan sigmoid function is a transfer function

W2 : Weight of the out layer and W1 : Weight of the input layer

Input : input (feature value) which need to be computed

b2 : bias of the output layer and b1 : bias of the hidden layer

This research mainly focused for detection of three diseases, which are Hawar Leaf, Anthracnose, and Pestalotiopsis Palmarum, that attack palm oil trees. All the three diseases could be detected based on the visual symptom of the tree.

In [10], the authors labeling a paper as "Choice of Efficient Image Classification Technique using Limited Device" to make available handy solution to farmers for come to a decision of the time of tomato harvesting by using Cellular phone with clustering technique for make use of less space and without requires any training for the Classifier. The captured tomato images were preprocessed, extracting the features and classifying the features using the classifier is clustering algorithms. They also describes clustering techniques does not requires any pre training the extracted features for classification because the clustering is unsupervised nature and it does not require more space to implemented on mobile devices. Their proposal comprises of 4 steps first one was Image representation, feature extraction and selection. The second steps was Set up similarity metrics suitable for special application. In step three involves Image clustering and finally form clusters based 
A Survey and Research Proposal of Plant Leaf Disease Diagnosis and Classification on Mobile..

on experts opinion and label it with abstract concepts. The proposal has been implemented using Android 2.0 and Java SDK.

III. Summarization Of The Existing Research Works

\begin{tabular}{|c|c|c|c|c|c|c|}
\hline Authors & 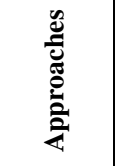 & $\begin{array}{l}\text { Plat } \\
\text { name }\end{array}$ & $\begin{array}{l}\text { Diseases / harvesting } \\
\text { identified }\end{array}$ & Features extracted & Classification/algorithm & Accuracy \\
\hline [2] Tasneem & 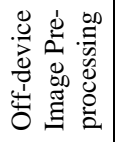 & Potato & $\begin{array}{c}\text { Early Blight and Late } \\
\text { Blight }\end{array}$ & Color, shape & $\begin{array}{l}\text { Leaf Vein Detection and } \\
\text { Blob detection algorithm }\end{array}$ & $94.1 \%$ \\
\hline $\begin{array}{l}\text { [3] B. Klatt et } \\
\text { al }\end{array}$ & \multirow{3}{*}{ 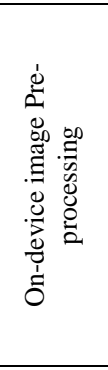 } & $\begin{array}{l}\text { Sugar } \\
\text { beet }\end{array}$ & $\begin{array}{c}\text { Cerospora beticola, } \\
\text { Ramularia, Phoma } \\
\text { betae }\end{array}$ & LBP & Naïve Bayer classifier & $97 \%$ \\
\hline $\begin{array}{l}\text { [4] Shovon } \\
\text { Paulinus } \\
\text { Rozario }\end{array}$ & & Paddy & $\begin{array}{l}\text { Bacterial Leaf Blight, } \\
\text { Brown Spot, Leaf } \\
\text { Blast, Leaf Scald }\end{array}$ & $\begin{array}{l}\text { blobs, area and } \\
\text { color }\end{array}$ & $\begin{array}{l}\text { Euclidean distance of } \\
\text { input and extracted images }\end{array}$ & Not given \\
\hline $\begin{array}{l}\text { [5]Shitala } \\
\text { Prasad et al }\end{array}$ & & Crop & $\begin{array}{c}\text { Powdery Mildew, } \\
\text { Downy Mildew, Late } \\
\text { Blight, Early Blight, } \\
\text { Tikka } \\
\end{array}$ & Gabor Wavelet & SVM with RBF & $98.96 \%$ \\
\hline $\begin{array}{l}\text { [7] Shitala } \\
\text { Prasad et al }\end{array}$ & 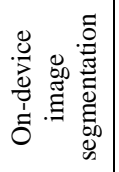 & Plant & $\begin{array}{l}\text { leaf spots and leaf } \\
\text { blotch }\end{array}$ & $\begin{array}{c}\text { K-means - } \\
\text { Segmentation. } \\
\text { GWT, GLCM- } \\
\text { features extraction }\end{array}$ & $\begin{array}{c}\text { Weighted k-Nearest } \\
\text { Neighbor }\end{array}$ & $93 \%$ \\
\hline $\begin{array}{l}\text { [8] Rahat Yasir } \\
\text { et al }\end{array}$ & \multirow{3}{*}{ 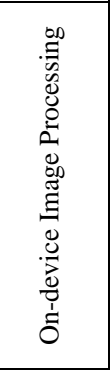 } & Crop & $\begin{array}{c}\text { brown leaf spot, } \\
\text { bacterial leaf blight, } \\
\text { brown spot, ufra and } \\
\text { rice blast }\end{array}$ & Color & $\begin{array}{l}\mathrm{YC}_{\mathrm{b}} \mathrm{C}_{\mathrm{r}}, \text { Histogram } \\
\text { algorithm }\end{array}$ & $85 \%$. \\
\hline [9] Alham F & & Palm Oil & $\begin{array}{l}\text { Hawar Leaf, } \\
\text { Anthracnose, and } \\
\text { Pestalotiopsis } \\
\text { Palmarum }\end{array}$ & $\begin{array}{c}\text { Median of RGB, } \\
\text { Quartile } 1 \text { of RGB, } \\
\text { Quartile } 3 \text { of RGB, } \\
\text { Standard deviation } \\
\text { of RGB, Shape }\end{array}$ & $\begin{array}{l}\text { Neural Network } \\
\text { Classification }\end{array}$ & $87.75 \%$ \\
\hline $\begin{array}{l}{[10] \text { Monika }} \\
\text { Bhatnagar et al }\end{array}$ & & Tomato & Harvesting & Not given & Clustering & Not given \\
\hline
\end{tabular}

\section{Research Proposal}

In early days, farmers required to conduct outsiders to mitigating diseases which is appeared in the plant leaves. When a disease strikes a plant, it needs immediate response to mitigation of the disease as soon as possible. For which the government had developed agricultural system, to act as a bridge between the farmers and agricultural scientists, experts, researchers and farmers. The developed system failed to meets the small and medium class farmers due to it required to implement in the fields of each farmer for their benefits. And the system does not respond fast enough for disease mitigation. To conquer the difficulty the government develop Agriculture data center which request the farmers to take the photograph of paddy leaves which is affected and to send to the center over the Internet. Then the personnel of the agriculture center processed the image and send the result to their mobile in the form of SMS or MMS. In these types of analysis has following problem.

1. Constant Internet connection is required to send the image which is expensive.

2. Rural areas are not able to connect over Internet.

3. Farmers felt difficult to operate this.

4. It is time consuming.

This present research would rectify all the above said problems. The objective of this research is to trace out the diseases in paddy leaf for the farmers mobile devices. By doing so, farmers can easily find out the disease and its causes and the effect. The farmer which he want to find the disease in the paddy leaf; just they only to take snapshot of the affected paddy leave use their mobile phone camera. Then the application, which is under developing and to be installed in mobile, is automatically preprocess the image for reduces noise in the image and uses K-means segmentation algorithm to segment the diseased portion of the image and extract the features from the segmented image using GWT algorithm. In this context a cluster analysis algorithm is 
developed for classifies the extracted features and store it to the database in order to diagnosis the disease in the plant leaf which is captured by the farmers. In case, any disease is found, the apps give the disease mitigation plan on their mobile phone itself. Otherwise, it exhibit no disease appear in the leaf. The developing application provides the interactive interface to the farmer's pocket to monitor and control the disease attack on the paddy leaf by anywhere-anyone- anytime. The architecture of a proposed research work is as following fig- 4.

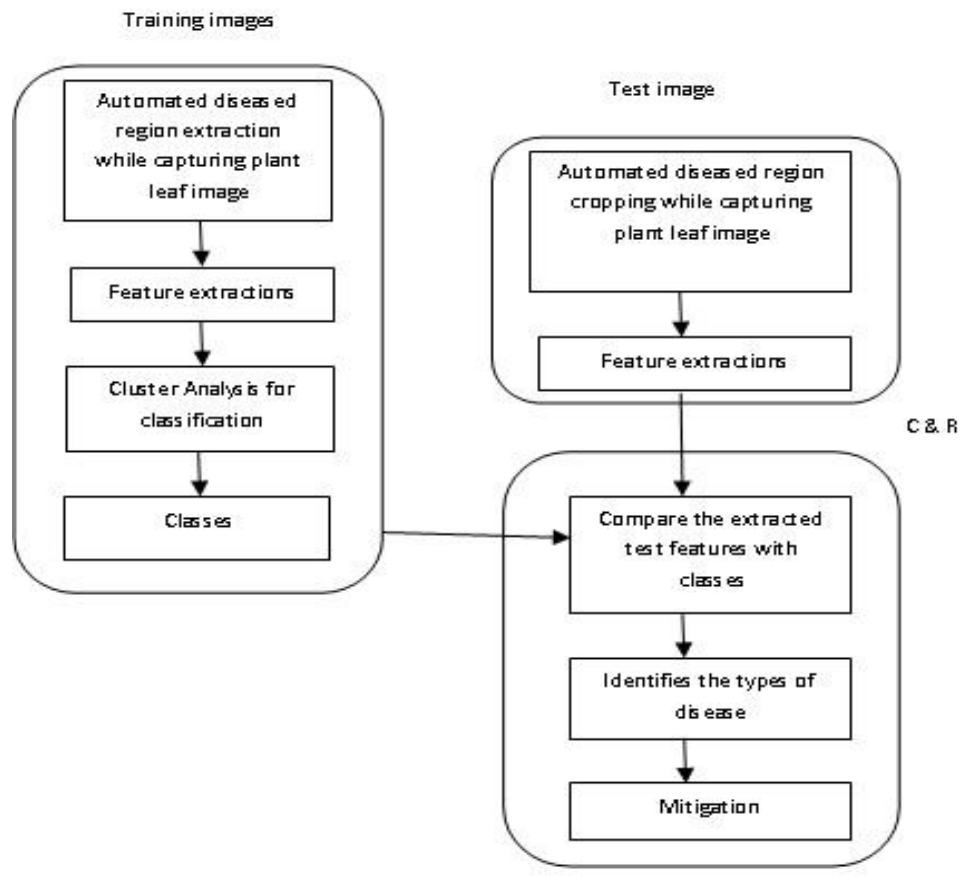

Fig -4

Some images which have been collected from the thanjavor district at ammaiyappan village. The images collected from three varieties such as 1009, ppt and 46; during the month of March 2015 which will be used to diagnosis and classification. Since the collected sample images which are illustrated as following table-I.

Table - I

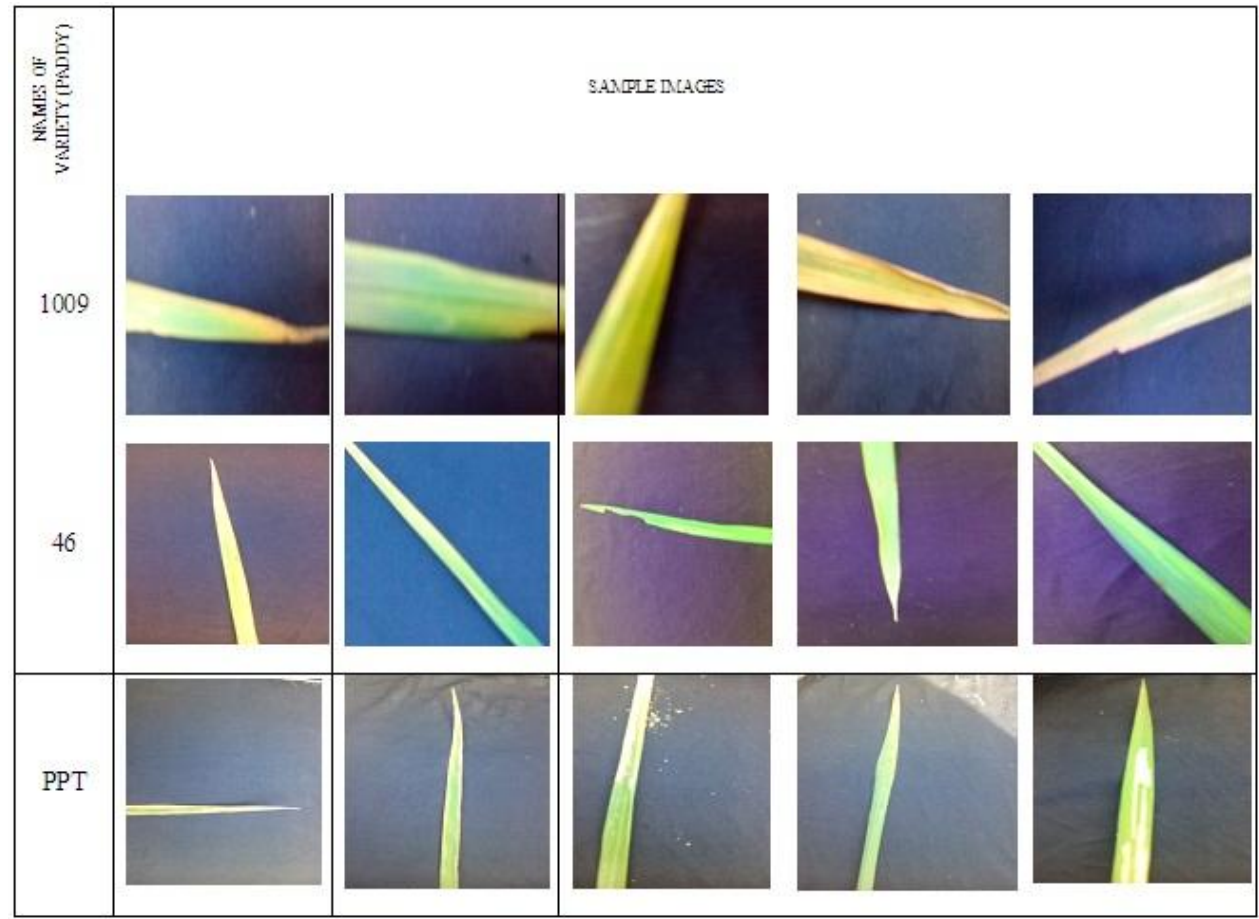




\section{Conclusion}

As this review, we can bring to a close that there are numeral traditions by which we can detect diseases in plant. Each one technique has various pros as well as restriction. This paper assess the techniques in data mining, image processing, artificial neural network, and etc., in use by researchers designed for detection, diagnosis and recognition of plant diseases on mobile devices. Still lot of research is going on for using various techniques to turn out automated plant diseases detection via mobile phone captured images. Our forthcoming research article will discover a cluster analysis algorithm for disease detection and classification for mobile devices with limited memory, higher processing speed and added accuracy.

\section{References}

[1]. Amy D. Timmerman and Kevin Korus - "Introduction to Plant diseases", the board of Regents of the University of Nebraska

[2]. Tasneem Tazeen Rashid Thuza Md. Sazzad Hossain - "Mobile Application for Determining Input Level Of Fertilizer And Detecting Diseases In Crops" - Thesis.

[3]. B. Klatt, B. Kleinhenz, C. Kuhn, C. Bauckhage, M. Neumann, K. Kersting, E.-C. Oerke, L. Hallau, A.-K. Mahlein, U. SteinerStenzel, M. Röhrig-"SmartDDS-Plant Disease Detection via Smartphone", EFITA-WCCA-CIGR Conference "Sustainable Agriculture through ICT Innovation", Turin, Italy, 24-27 June 2013.

[4]. Shovon Paulinus Rozario- " Krishokbondhu - An automated system for diagnosis of paddy disease, Thesis, SCHOOL OF ENGINEERING AND COMPUTER SCIENCE, Department of Computer Science and Engineering, BRAC University, Submitted on September 1, 2014

[5]. Shitala Prasad, Sateesh K. Peddoju and Debashis Ghosh - " AgroMobile: A Cloud-Based Framework for Agriculturists on Mobile Platform", International Journal of Advanced Science and Technology Vol.59, (2013), pp.41-52 http://dx.doi.org /10.14257/ijast.2013.59.04 ISSN: 2005-4238 IJAST Copyright (C)2013 SERSC.

[6]. S.A. Ramesh Kumar etc., al. -“A Novel and High Speed Technique for Paddy Crops Disease Prediction in Wireless TeleAgriculture Using Data Mining Techniques”, Middle-East Journal of Scientific Research 22 (9): 1430-1441, ISSN 1990-9233, (C) IDOSI Publications, 2014.

[7]. Shitala Prasad - Sateesh K. Peddoju - Debashis Ghosh - "Multi-resolution mobile vision system for plant leaf disease diagnosis", Received: 16 December 2013 / Revised: 17 September 2014 / Accepted: 31 January 2015 @ Springer-Verlag London 2015

[8]. Rahat Yasir and Nova Ahmed- "Beetles: A Mobile Application to Detect Crop Disease for Farmers in Rural Area", Workshop on Human and Technology, 8 - 10 March 2014, Khulna, Bangladesh.

[9]. Alham F. Aji, Qorib Munajat, Ardhi P. Pratama, Hafizh Kalamullah, Aprinaldi, Jodi Setiyawan, and Aniati M. Arymurthy- “ Detection of Palm Oil Leaf Disease with Image Processing and Neural Network Classification on Mobile Device “, International Journal of Computer Theory and Engineering, Vol. 5, No. 3, June 2013.

[10]. Monika Bhatnagar , Dr. Prashant Kumar Singh - "Choice of Efficient Image Classification Tehcnique using Limited Device", International Journal of Electronics and Computer Science Engineering 1070 Available Online at www.ijecse.org.. 